\title{
Modelling of Surfaces of Engineering Products on the Basis of Array of Points
}

\author{
Yevgen HAVRYLENKO, Jose I. CORTEZ, Yuliia KHOLODNIAK, Hanna ALIEKSIEIEVA*, Gregorio T. GARCIA
}

\begin{abstract}
The method of designing elements of the surfaces' frames based on array of points is suggested in the work. Elements of frames are contours that are received via interpolation of sets of points, which are selected from the initial array of points. The algorithms have been developed for design plane and spatial contours that represent the curves with specified geometrical properties with prescribed accuracy. Formed contours are used as elements of «Profile» and «Guide Curves» at forming the model of surface by means of function of «Lofted Surface» in CAD system. Using the method of designing elements of frames of the surfaces is actual for modeling of surfaces of technical items that function-interact with the environment. The developed method was proven while modelling functional surfaces that bound an impeller blade channel of a turbine compressor.
\end{abstract}

Keywords: $C A D$; design engineering; fitting; interpolation; reverse engineering; smoothing methods; surface fitting

\section{INTRODUCTION}

Nowadays industrial development involves the use of different CAX systems for the design of new products, and for reverse engineering. The task of reverse engineering is to create a copy of the existing manufacturing item. Reverse engineering implies technologies which allow recreating shape and properties of the real physical entity [1].

A necessary step in reverse engineering or designing new products is the creation of a CAD model. The use of CAD systems leads to the rapid introduction of new products on the market. Features of the use of CAD systems in mechanical engineering were analyzed in [2].

The works [3-4] show examples of product development using the SolidWorks CAD system. Development and virtual testing of CAD models of products can reduce the time of implementation of new products and reduce the cost of technical preparation of production.

When solving reverse engineering problems, the initial data is the array of points belonging to the surface of the item. Point data of the array are obtained via 3D-scanning of the physical entity. Shortage of information about design of the item results in deviation of the surface of the model which is created on the basis of array of points, from the surface of the initial item [5-7]. The accuracy of representing initial item is the main criterion of the quality of the achieved solution. Minimization of error allows avoiding the need for calculations in respect of design and mechanical article strength.

The task of ensuring required design accuracy may be regarded as solved if the maximal deviation of the model from the initial surface does not exceed the accuracy with which numerical control tool (NCT) provides machining of the command paths [8].

The requirements to providing accuracy of reverse engineering are increased while designing items whose function is to interact with the environment. The main requirement to the surfaces of such items is to provide laminar character of their environment flow [9].

Such surfaces are called the surfaces with enhanced dynamic properties or dynamic surfaces. Enhanced dynamic properties are required for the surfaces that bound casewoods in aircraft, motor car construction, shipbuilding, blades of turbines, internal combustion engine channels, hydraulic hookups, operative parts of agricultural machinery. Constant rise in quality standards on modelling of such surfaces is caused by increase of speed of interaction with the environment [10].

At evaluating engineering product functional properties the dynamic properties of the surface are defined with stream conditions of the environment. The stream appears in a boundary layer lengthwise the surface. The main requirement is a regular, stable, scheduled character of flow. Deviation from the initial geometric characteristic of the item results into onset of turbulence-turbulence inside the flow.

In CAD system the model of the surface is generated on the basis of the frame [11-12]. Frame elements are contours that are received via interpolation of sets of points. For the time being the most developed are the methods that design contours on the basis of analytically specified curves. The curve is being formed, which consists of analytically set pieces. It traverses the set of points, providing specified characteristics of the contour in these points. Up-to-date CAD packages use exactly such techniques for designing of contours.

The limitation of the contours designing based on the analytically set curves is that expansion of conditions imposed on the contour requires increase of parametric number of curves that form its pieces. Meanwhile, specific points inevitably appear. The term "specific points" means the points of change of trend of the curve, points of inflection and self-intersection or points of variation of growth-decrease of curvature and torsion along the curve. Uncontrollable appearance of specific points lowers the quality of obtained solution:

- the deviation is increased, with which the model represents the initial surface;

- violation of the set conformity of variation of the geometric characteristics along the lines that are used as linear elements of the frame of the surface.

It is especially important to control the appearance of specific points at modelling the dynamic surfaces.

The process of item manufacturing includes the following stages:

- Frame of the surface is generated on the basis of initial array of points-set of the curves belonging to the surface: 
- $\quad$ subsets are distinguished from the array of points-sets of points. The term «set of points» is understood as an ordered set of points, that can be interpolated with a plane or space curve;

- as a result of interpolation of the sets of points the elements of the frame are generated-contours consisting of the segments of the curves that are smoothly joined in the initial points;

- The model of surface of the item is designed on the basis of the frame in CAD system.

Control program on machining the item with the numerical control tool is created in CAM-system. The reference data for creating control program is a model which is derived in CAD system.

\section{ANALYSIS OF RECENT STUDIES AND PUBLICATIONS}

The existing methods of designing contours on the basis of analytically specified functions (methods of continuous geometric simulation) include the methods of global modelling and methods of piecewise smooth approximations. The methods of global modelling define the geometric image with one equation. In works $[13,14]$ the contour is described with Newton and Hermite algebraic polynomials. When designing contours via mentioned methods the degree of equation of interpolating curve grows at increasing the number of initial points. With increasing the degree of the equation the parametric number of the curve grows up. This results in uncontrollable appearance of specific points. For the polynomial curve these are first of all points of inflection. The considered methods cannot be used for generation of elements of dynamic surfaces' frames.

It is possible to avoid uncontrollable appearance of the inflection points (appearance of oscillation) applying the method of interpolation on the basis of trigonometric functions. Such a method was developed in the work [15]. The limitation of this method is that it is calculated for interpolation of small quantity of points. This peculiarity of the method decreases the range of its practical application.

The method of contours design via pieces of secondorder curves ensures absence of oscillation at interpolating any quantity of initial nodes. This method is suggested in the works [16]. It provides for single position of the tangent line to contour in the connection points of its pieces. Using of second-order curves guarantees an absence of oscillation lengthwise the contour pieces. The limitation of the method is violation of regularity of curvature values in the connection points of contour pieces.

The method that was developed in the work [17] allows ensuring regularity of curvature values lengthwise the contour. This method provides for using Bézier Curves of the third or high orders. The main drawback of contours design based on Bézier splines is that they do not provide for locality of the curve shape's controlling on the pieces of the contour.

The methods of contours design based on B-splines and NURBS curves are developed in the works [18-20]. Using of B-spline or NURBS curve increases maximal locality of the curve shape's monitoring as compared to other methods of continuous geometric simulation. Locality of controlling the shape of B-spline is limited.
Amount of areas of B-spline, which simultaneously change the form at correcting per unit is more than the degree of its equation. Additional conditions that are put on B-spline require the increase of degree of equation. Such conditions are setting of the fixed positions of tangents and values of curvature in the key points.

Local adjustment of shape allows providing the monotonous change of curvature along B-spline at interpolation of small amount of initial points. It is impossible to ensure regular variation of curvature lengthwise the odd-shaped contours that consist of a big number of pieces that are generated with B-spline or NURBS curve.

If a dynamic surface is designed on the basis of frame, the properties of surface are provided by properties of linear elements-the plane and space curves. From the geometrical point of view the enhanced dynamic properties of the surface can be provided due to the regular change of curvature and torsion along the elements of the frame. The regular change of characteristics of the curve should be understood as monotonous growth or decrease of their values along the pieces of curve. Thus the amount of monotonous pieces must be minimum. The drawbacks of the methods of continuous geometric simulation limit their possibilities on generating contours, along which the values of geometric characteristics change regularly.

The purpose of the research is to develop a method of designing the frame of the dynamic surfaces on the basis of specified number of sets of points. To achieve this purpose it is necessary to solve the following tasks:

- develop the method of analysis of initial set of points, allowing determining pieces in order to be able to be interpolated with a monotonous curve. We will name a smooth curve of permanent motion, along which the values of curvature monotonously increase or decrease, a monotonous curve;

- develop the algorithm of designing the plane smooth contour with a regular monotonic variation of curvature;

- develop the algorithm of space smooth contour's designing with a regular variation of curvature and torsion's values;

- test the developed method for modelling functional surfaces of the turbine compressor's impeller of an axialradial type.

\section{RESULTS AND DISCUSSION}

Consider the method of modelling the frame of the dynamic surfaces proposed in this article. The method that we developed was applied to design a model of working surfaces that bound the blade channel of a turbine compressor.

\subsection{The Modelling Method of One-Dimensional Contours}

The method we are developing presupposes designing of one-dimensional contours based on the area of their possible positioning. This area is estimated based on known geometric properties of the contour.

The contour is designed in a form of a discretely presented curve (DPC) which is represented as an ordered set of points and geometric characteristics of the curve. 
In the process of DPC's design the set of points is generated. It consists of arbitrary large number of points [21-23]. These are reference points and points of condensation. Positions of points of condensation are identified on the generated curve.

The set of points is analyzed before the condensation. It results in determining the pieces on whose basis the curve with a monotonic curvature and torsion variation can be generated. Received pieces are generated separately and joined to each other. Positions of the points of condensation are defined on the basis of initial points' positions and requirements that are imposed on DPC. Such conditions are absences of oscillation, a regularity of values of the curvature and torsion, a monotonous change of the radii of curvature lengthwise the curve.

The radii of curvature in the points of plane DPC are estimated via radii of curvature of quadratic Bézier Curve $\left({ }^{\prime} R_{i}, R_{i}^{\prime}\right)$. The control points of Bézier Curve coincide with the vertexes of a basal triangle (BT). BT is limited with the tangent to DPC lines passing through sequential points and the chord that connects these points (Fig. 1). Fig. 1 to Fig. 6 are the authors' own work.

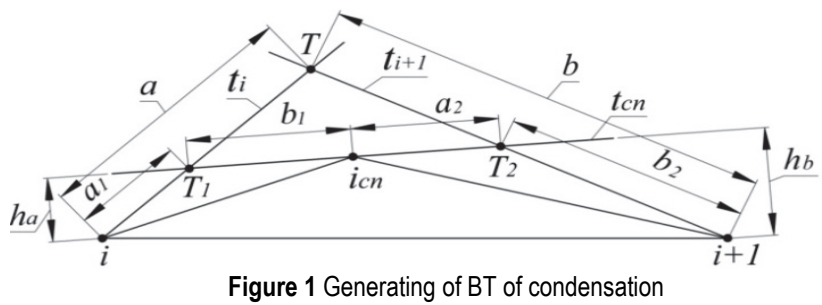

The values of the radii of curvature in the points $i$ and $i+1$ are estimated by the equation:

${ }^{\prime} R_{i}=\frac{a^{3}}{S}, R^{\prime}{ }_{i+1}=\frac{b^{3}}{S}$

where $a=|i ; T|$ and $b=|T ; i+1|$ are the lateral lengths of $B T(i, T, i+1) ; S$ is an area of BT.

The positioning of the condensation point $\left(i_{c n}\right)$ and a tangent line to the contour in this point $\left(t_{c n}\right)$ is assigned inside of the initial BT. As a result we get two BTs of condensation $\left(i, T_{1}, i_{c n}\right)$ and $\left(i_{c n}, T_{2}, i+1\right)$.

The scheme of condensation of a set of points allows providing assigned values of the radii of curvature in the points of DPC. Thereto the points of condensation assigned inside of BT are assigned so that the values of the radii of curvature $R_{i}^{c n}$ and $R_{i+1}^{\prime^{c n}}$ in the points $i$ that determine BT of condensation equal the values of $R_{i}$ and $R_{i+1}$ assigned in these points. That is ' $R_{i}^{c n}=R_{i}$ and $R^{\prime c n}{ }_{i+1}^{c n}=R_{i+1}$. Taking into account the parameters of BT this condition is of the form [16]:

$\frac{a^{3}}{S}=\frac{a_{1}^{3}}{S_{1}}$ and $\frac{b^{3}}{S}=\frac{b_{2}^{3}}{S_{2}}$

where $a_{1}=\left|i ; T_{1}\right|$ and $b_{2}=\left|T_{2} ; i+1\right|$ are the lateral lengths of $B T\left(i, T_{1}, i_{c n}\right)$ and $B T\left(i_{c n}, T_{2}, i+1\right)$ respectively; $S_{1}$ and $S_{2}$ are the areas of the specified BTs.
In order to provide the regularity of variation of the curvature values it is necessary to control the values of ' $R_{c n}$ and $R_{c n}^{\prime}$, to be equal; they determine $B T\left(i, T_{1}, i_{c n}\right)$ and $B T\left(i_{c n}, T_{2}, i+1\right)$ in a common point. In consideration of parameters of BT this equation is of the form

$\frac{b_{1}^{2}}{a_{2}^{2}}=\frac{h_{a}}{h_{b}}$

where $h_{a}$ and $h_{b}$ are the distances from the points $i$ and $i+$ 1 to the tangent line $t_{c n}$.

Assignation of the positions of the tangent lines in the initial points according to the Eq. (3) allows generating a contour after each step of condensation as a compound curve from the arcs of Bézier Curves that are connected to provide common osculating circle in the connection point. Meanwhile, each Bézier Curve piece which is limited with the sequential points can have a piece of increase and a piece of decrease of the radii of curvature.

Fulfillment of the conditions ' $R_{i}^{c n}<R_{c n}$ a nd $R_{c n}^{\prime}<R_{i+1}^{\prime c n}$, is controlled in the process of condensations. These conditions are expressed via parameters of BT like this $a_{1}<b_{1}$ and $a_{2}<b_{2}$.

Implementation of specified conditions provides position of tangents of lines in initial points at which forming of monotonous DPC is possible.

Thus, fulfilment of the conditions of Eq. (2), Eq. (3) and Eq. (4) in the process of sequential condensations provides forming of sequence of BT. Got BTs limit the area of possible location of the monotonous curve which interpolates the set of points.

Simultaneously with forming of DPC we get the contour from the pieces of Bézier curves. The contour presents a monotonous curve with an error, which does not exceed the height of BT.

The space DPC is formed on the basis of a set of points, which does not belong to one plane. Every three sequential points define a plane. We will call them superimposed planes (SP). Let's require that the angle between adjacent SPs $\left(\varphi_{i}\right)$, inside of which there is a piece of DPC, should not exceed 180 degrees. Then the direction of rotation of SP is relative to the chord $(i ; i+1)$ at the angle $\varphi_{i}$ till the alignment with $S P(i, i+1, i+2)$ coincides with the direction of the trend of DPC (Fig. 2).

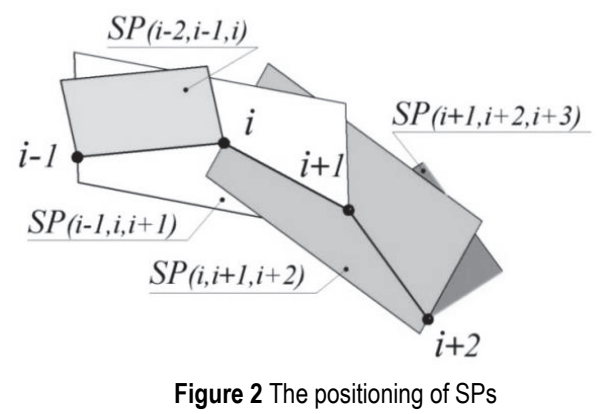

Four sequential SPs through the points $i$ and $i+1$ bound a tetrahedron. This tetrahedron is the area of possible positioning of DPC of constant trend on the piece $i, \ldots, i+1$. 
The chain of tetrahedrons that are defined in all pieces is the region of positioning of smooth curve line of constant trend that interpolates the initial set of points (Fig. 3).

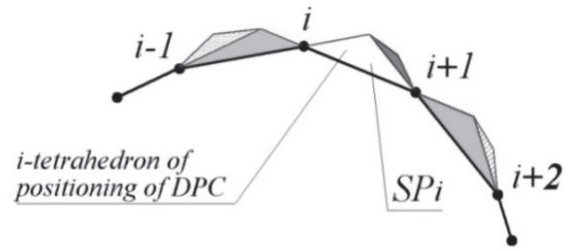

Figure 3 The area of positioning of DPC of constant trend

The tangent to DPC line $\left(t_{i}\right)$ is located inside two adjacent dihedral angles: $\varphi_{i}$ and $\varphi_{i+1}$. The position of the tangent $t_{i}$ is determined with the planes that touch DPC in the point $i$ and traverse antecedent $(i-1)$ and following $(i$ +1 ) initial points (planes of $T P\left(i-1, t_{i}\right)$ and $T P\left(t_{i}, i+1\right)$ correspondingly) (Fig. 4).

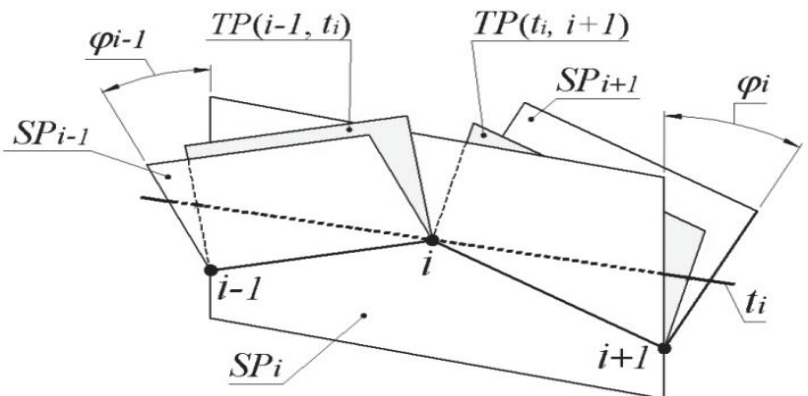

Figure 4 Estimation of the position of the tangent straight lines and planes

Positions of the tangent planes are assigned on the basis of the values of discrete torsion on the pieces of DPC that are defined with the properties of the initial set of points. To a first approximation the torsion on the piece $i$, $\ldots, i+1$ will be estimated with the value $B_{i}^{\varphi}=\frac{3 \varphi_{i}}{h_{i-1}+h_{i}+h_{i+1}}$, where $\varphi_{i}$ is the angle between the adjacent superimposed planes; $h_{i}=|i ; i+1|$ is the length of the line.

After assigning the tangent planes the discrete torsion on i-piece of DPC is estimated with the formula $B_{i}^{\psi}=\frac{3 \psi_{i}}{h_{i}}$, where $\psi_{i}$ - angle between $T P\left(t_{i}, i+1\right)$ and $T P(i$, $t_{i+1}$ ) (Fig. 5).

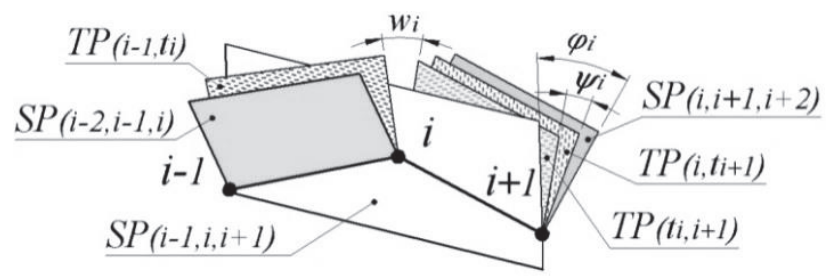

Figure 5 Range of positioning the osculating plane

Value of the angle $\psi_{i}$ is assigned on the basis of the condition $B_{i}^{\varphi}=B_{i}^{\psi}$.

After assigning tangent planes, another criterion will be got for estimating the torsion on the pieces of DPC
$B_{i}^{w}=\frac{3 w_{i}}{h_{i-1}+h_{i}}$, where $w_{i}$ - angle, limited to the planes $T P(i$ $\left.-1, t_{i}\right)$ and $T P\left(t_{i}, i+1\right)$.

Positioning the tangent planes inside the angles $\varphi_{i}$, limited with adjoining planes is determined by simultaneous implementation of conditions (5) and (6).

Planes $T P\left(i-1, t_{i}\right)$ and $T P\left(t_{i}, i+1\right)$ that touch DPC in one point bound the range $w_{i}$ (Fig. 5) of possible positioning of the osculating plane $\left(O P_{i}\right)$. The position of $O P_{i}$ is assigned in order to divide angle $w_{i}$ at the angles ' $w_{i}$ and $w_{i}^{\prime}$ in correlation $\frac{w_{i}}{w_{i}{ }^{\prime}}=\frac{\psi_{i-1}}{\psi_{i}}$

The lengthwise DPC values of torsion grow monotonically while generating DPC, the position of tangent planes has to provide for fulfillment of conditions $\ldots<B_{i-1}^{\psi}<B_{i}^{w}<B_{i}^{\psi}<B_{i+1}^{w}<\ldots$.

Assignation of tangent and osculating planes is specified with the tetrahedron of positioning of DPC. It results in a new chain of tetrahedrons. Its facets belong to the tangent and osculating planes. This chain of tetrahedrons is an area of possible positioning of smooth DPC of constant trend. The positions of the main trihedrons are assigned in its nodes.

The point of condensation is assigned inside the tetrahedron of DPC's positioning. The initial range of positioning of the point $i_{c n}$ on the piece $i, \ldots, i+1$ is a segment $\left(T_{i} ; T_{i+1}\right)$. It belongs to the straight line of intersection of the tangent planes $T P\left(t_{i}, i_{c n}\right)$ and $T P\left(i_{c n}, t_{i+1}\right)$ (Fig. 6).

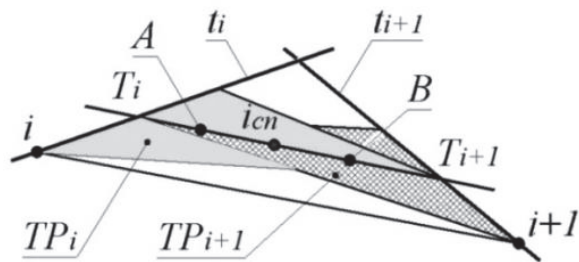

Figure 6 Estimation of the range of positioning of the condensation point

Assignment of $i_{c n}$ in the range of the segment $\left(T_{i} ; T_{i+1}\right)$ provides for the assigned behaviour of values of discrete torsion lengthwise DPC, which is derived as a result of condensation. The specific position of $i_{c n}$ is estimated on the basis of monotonic variation condition of the radii values of curvature lengthwise DPC.

While generating DPC, lengthwise which the radii of curvature grow monotonically, the condition has to be fulfilled $\ldots<R(i-1, i, i+1)<R(i, i+1, i+2)<\ldots$ where $R(i-1, i, i+1)$ is a radius of the superimposed circle that traverses the points $i-1, i, i+1$.

The position of condensation point on the piece of DPC with monotonic growth of radii of curvature is assigned on the basis of the condition $R\left(i-1, i, i_{c n}\right)<R(i$, $\left.i_{c n}, i+1\right)<R\left(i_{c n}, i+1, i+2\right)$.

The points of condensation are assigned sequentially inside the maximum size tetrahedron of positioning of DPC. Fulfilment of the specified conditions at assigning every point of condensation provides for generating the points set that belongs to DPC with a regular monotonic variation of radii of curvature and torsion. 
DPC is considered to be generated when the area of its possible positioning does not exceed prescribed accuracy of designing the contour [24]. For plane DPC it is a base triangle, and for spatial DPC it is the tetrahedron of its possible location. Thereafter, the contour can be generated as a concomitant broken line, torispherical line of the circle, pieces of Bézier curves or B-spline, that interpolate the derived set of points. Generated contour represents a curve with the set geometrical properties with accuracy which does not exceed the area of possible location of this curve.

The general feature of forming of plane and spatial contours via the offered method is an analysis of initial set of points. As a result of analysis the areas are determined that are possible to be interpolated with the curve with a monotonous change of geometrical characteristics, and also pieces where specific points are inevitable. The method allows generating plane and spatial DPCs, where the number of specific points is minimal.

Control of the error with which generated contour presents the set curve is a feature and advantage of our method. In case of interpolation of initial set of points by the analytically set curves [16-19], the error of interpolation is not controlled.

Another feature of our method is providing monotonous change of curvature and torsion's values along DPC. The familiar methods of contours' forming do not provide control of these characteristics along the curve. For example, adjustment of B-spline by means of setting polygon allows controlling the convexity of the plane curve and movement of the space curve. Control of monotony of geometrical characteristics' change along DPC allows decreasing 2 - 3 times the error of its replacement with the contour consisting of the continuous curves.

\subsection{Designing Working Surfaces of Products}

The blade channel is bounded with the surfaces of the impeller and turbocharger housing.

Necessity for creation of models in CAD system is caused by the decision of top management of the enterprise that ordered their development, about the independent manufacture of the impeller with the use of machine-tools with numerical control. Before, the impellers were bought from other enterprises. Basic data about the design of the impeller are taken from the engineering drawing, which contains information necessary for quality control of the finished product.

Design quality is determined by implementation of the enterprise's requirement: the productivity of the item, manufactured with the use of new technology, must exceed the productivity of initial item.

The surfaces that bound the blade channel have to provide for fulfilment of the requirements [25-26]:

- laminar character of the surfaces' flow of the channel with gas stream;

- gas pressure increase lengthwise the blade channel;

- change of the flow direction from the axial in the channel entrance to the radial in the channel outlet.

The required properties of the flow are provided with the geometric characteristics of working surfaces and parameters of the blade channel.
The required functional properties of working surfaces of a turbine compressor are provided with the geometric properties of the elements of the frame specifying the surface. These properties are a regular variation of curvature and torsion's values at minimal under the conditions of the problem number of singular points of the curve: points of inflection, change of direction of increasing the values of curvature and torsion lengthwise the curve.

The impeller is manufactured in foundry and is a nave with blades. The reference data for designing a geometrical model of the impeller is a drawing of the nave and positions of 154 points, belonging to the surface of the blade. Positions of these points are set with the initial drawing [27].

The model of the blade's surface is designed on the basis of the frame that consists of 22 plane sections and two spatial guide curved lines (Fig. 7a).

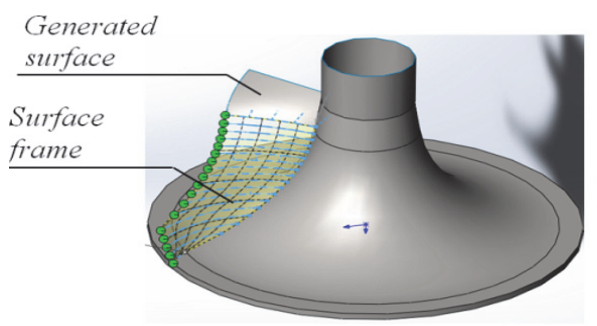

a)

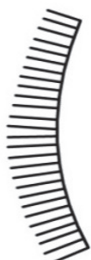

b)

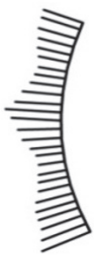

Figure 7 Design of the frame of the blade surface

Every flat section is formed on the basis of 7 points, abstracted from the initial array of points. One of the horizontal sections of the blade surface is represented in Fig. 7b. An error with which an initial set of points presented the curve with a regular change of curvature does not exceed $10^{-2} \mathrm{~mm}$. Condensation of the set of points results in receiving 58 points specifying horizontal section of the blade. An error with which the received set of points presents a curve with a regular change of curvature $\left(\delta_{i}\right)$, makes from $2 \cdot 10^{-4} \mathrm{~mm}$ to $8 \cdot 10^{-4} \mathrm{~mm}$. The points of the derived DPCs are interpolated with B-splines that are used as linear elements of the surface model in SolidWorks.

Fig. $7 \mathrm{~b}$ represents the curve of curvature variation lengthwise the horizontal section that is generated with Bspline that interpolates the points set consisting of 58 nodes. It is derived via our method. To compare with, Fig. $7 \mathrm{c}$ represents the curve of curvature variation lengthwise the section that is generated with B-spline that interpolated the points set consisting of 7 nodes.

Application of our method made it possible to decrease the derived DPCs which are interpolated with a nonperiodic cubic B-spline. Thereafter, the elements of the frame are imported into CAD system of SolidWorks and the model of the working surface of the blade is obtained via function «Surface on the sections» of CAD system.

Using our method made it possible to decrease the number of points of trend change of curvature growth lengthwise the curve from 3 at B-spline that interpolates the initial points set (Fig. $7 \mathrm{c}$ ) to 1 at B-spline that interpolates generated set of points (Fig. $7 \mathrm{~b}$ ).

Guide lines of the frame are generated on the basis of the sets of points located on the nave and blade edge. Spatial right-hand DPCs are generated on the basis of the 
reference set of points. The radii of the osculating circles are monotonically growing lengthwise these DPCs.

DPCs are received as a result of three successive condensations of the initial set of points, consisting of 22 points. The generated set of points consists of 169 points. Tab. 1 presents discrete characteristics of the received DPC that are determined in the initial points.

\begin{tabular}{|c|c|c|c|c|}
\multicolumn{5}{|c|}{ Table 1 The discrete characteristics of the set of points } \\
\hline$i$ & $i_{c n}$ & $\begin{array}{c}h_{i}=|i, i+1| / \\
\mathrm{mm}\end{array}$ & $\begin{array}{c}B_{i}^{w} \times 10^{-2} / \\
\mathrm{rad} / \mathrm{mm}\end{array}$ & $R(i-1, i, i+1) / \mathrm{mm}$ \\
\hline 1 & 1 & 3.36017 & 0.06232 & 800.54876 \\
\hline 2 & 9 & 3.34545 & 0.08621 & 611.65891 \\
\hline 3 & 17 & 3.33075 & 0.12654 & 305.65891 \\
\hline 4 & 25 & 3.31605 & 0.15002 & 222.36874 \\
\hline 5 & 33 & 3.30135 & 0.16365 & 150.24895 \\
\hline 6 & 41 & 3.28667 & 0.18654 & 121.658942 \\
\hline 7 & 49 & 3.27199 & 0.21986 & 116.956214 \\
\hline 8 & 57 & 3.25732 & 0.24002 & 115.865234 \\
\hline 9 & 65 & 3.24267 & 0.25647 & 115.000211 \\
\hline 10 & 73 & 3.22801 & 0.27641 & 114.401254 \\
\hline 11 & 81 & 3.21337 & 0.28643 & 114.013265 \\
\hline 12 & 89 & 3.19874 & 0.29873 & 113.562480 \\
\hline 13 & 97 & 3.18412 & 0.30002 & 112.956841 \\
\hline 14 & 105 & 3.16950 & 0.32001 & 112.256148 \\
\hline 15 & 113 & 3.15489 & 0.35458 & 112.000124 \\
\hline 16 & 121 & 3.14029 & 0.38647 & 118.658421 \\
\hline 17 & 129 & 3.12571 & 0.40726 & 156.654182 \\
\hline 18 & 137 & 3.11113 & 0.42002 & 188.325874 \\
\hline 19 & 145 & 3.09656 & 0.42158 & 241.695241 \\
\hline 20 & 153 & 3.08201 & 0.46972 & 284.265147 \\
\hline 21 & 161 & 3.06746 & 0.51482 & 305.215964 \\
\hline 22 & 169 & - & 0.55624 & 321,541362 \\
\hline
\end{tabular}

Tab. 2 gives discrete characteristics of the received DPC in the points of condensation, which are generated on a piece between initial points 12 and 13. After condensing of the whole set of points, the numbers of the indicated initial points turned into 89 and 97, accordingly. Maximal absolute error of forming monotonous DPC $\left(\delta_{i}\right)$ on the piece 89 - 97 does not exceed $4.94 \times 10^{-4}$.

Table 2 The discrete characteristics at condensation points

\begin{tabular}{|c|c|c|c|c|c|}
\hline \multicolumn{7}{|c|}{ Table 2 The discrete characteristics at condensation points } \\
\hline & $h_{i} / \mathrm{mm}$ & $\begin{array}{c}B_{i}^{w} \times 10^{-2} \\
/ \mathrm{rad} / \mathrm{mm}\end{array}$ & $\begin{array}{c}B_{i}^{\varphi} \times 10^{-2} \\
/ \mathrm{rad} / \mathrm{mm}\end{array}$ & $\begin{array}{c}R(i-1, i, i+ \\
1) / \mathrm{mm}\end{array}$ & $\begin{array}{c}\delta_{i} \times 10^{-4} / \\
\mathrm{mm}\end{array}$ \\
\hline 89 & 0.41556 & 0.29873 & 0.29883 & 113.32153 & 4.93956 \\
\hline 90 & 0.41531 & 0.29889 & 0.29890 & 113.25842 & 4.88954 \\
\hline 91 & 0.41506 & 0.29891 & 0.29916 & 113.16854 & 4.76584 \\
\hline 93 & 0.41481 & 0.29906 & 0.29915 & 113.08651 & 4.62581 \\
\hline 94 & 0.41456 & 0.29921 & 0.29936 & 113.01548 & 4.52178 \\
\hline 95 & 0.41432 & 0.29954 & 0.29968 & 112.99541 & 4.36851 \\
\hline 96 & 0.41407 & 0.29996 & 0.29998 & 112.92653 & 4.28457 \\
\hline 96 & 0.41584 & 0.30000 & 0.30000 & 112.87542 & 4.12018 \\
\hline 97 & 0.41382 & 0.30002 & 0.30004 & 112.81530 & - \\
\hline
\end{tabular}

After forming working surfaces of impeller blades, the model of blade channel was created. This channel is limited to the surfaces of impeller, which limit blades and nave, and also the surface of the cover.

Models of the nave and cover are obtained via functions of CAD system of SolidWorks "Revolved surface". The line of motion, which sets the surface of rotation, is specified in the window of "Property Manager". It is a curve consisting of arcs, two circles, the parameters of which are taken from the detailed drawing.

The gas-dynamic analysis of the blade channel is performed after designing the geometrical model. Analysis was carried out via module of Solid Works Flow
Simulation. The reference parameters of the flow are: inlet pressure $(8.1 \mathrm{kPa})$ and outlet pressure $(170 \mathrm{kPa})$, inlet flow velocity $(90 \mathrm{~m} / \mathrm{s})$ and outlet flow velocity $(205 \mathrm{~m} / \mathrm{s})$.

Analysis of the reference model of the impeller revealed that a flow movement pressure in the mid portion of the blade channel decreases by $11 \%$ (Fig. 8). The pressure decrease can be caused with the appearance of the turbulence in the flow [10].

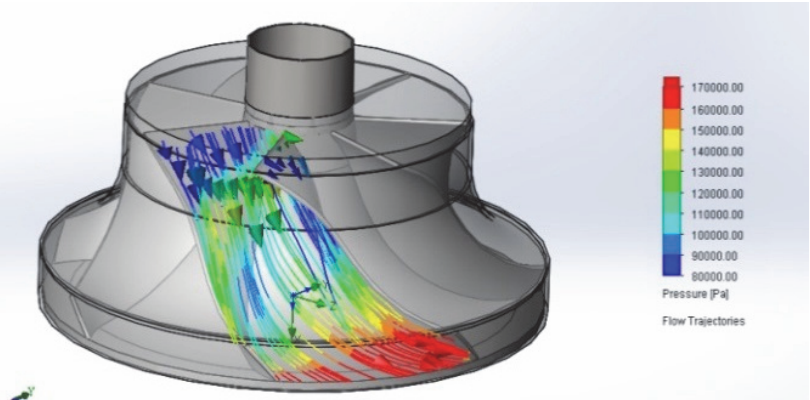

Figure 8 Distribution of pressure inside the flow in the blade channel

In order to avoid turbulence the surface that bounds the channel has been corrected. The correction is made to optimize the graph of the channel normal sections areas.

The frame, setting an optimized surface of the blade channel, consists of axial guide line and family of normal sections. The space curve of permanent motion, not containing special points, is used as an axial line. The basic requirement to the normal sections is a monotonous change of their areas along an axial line [28].

The reference data for generating the axial line of the channel are current streamlets that pass through the mass centre of the inlet and outlet sections. Current streamlets are obtained automatically via functions of FlowWorks. The generated initial points set specify the axial line. The set consists of 9 nodes. The initial points are assigned to the pieces that join points that divide current streamlets equally (Fig. 9).

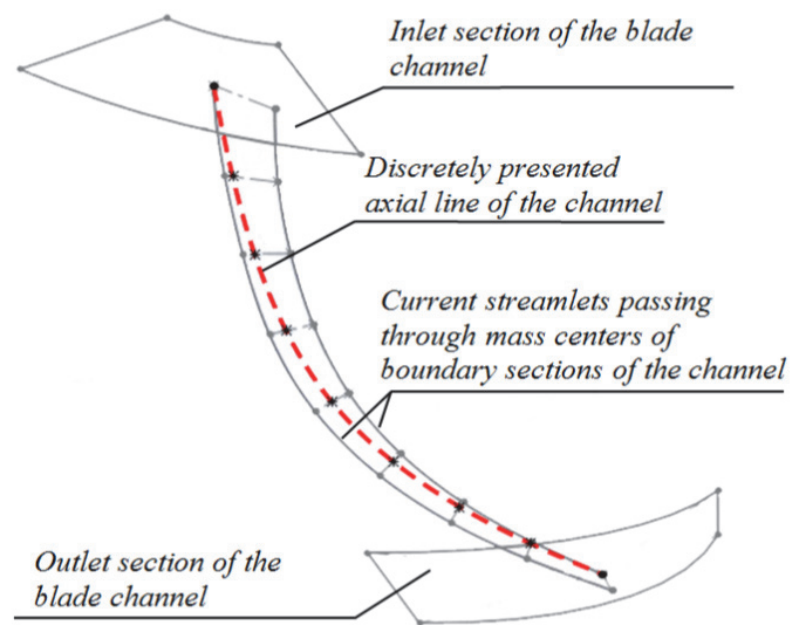

Figure 9 Generating the axial line of the blade channel

DPC has been generated on the basis of the initial points. This DPC of a constant trend does not contain specific points and consists of 72 nodes.

The positions of DPC normal planes that determined inlet, outlet and 7 intermediate channel sections are determined in 9 points, uniformly distributed lengthwise 
the generated DPC. Normal planes are set by passing through the given point athwart tangentline to the DPC in this point. Normal planes cross the surface of initial blade channel on 9 normal sections: inlet, outlet and 7 intermediate sections of the channel.

By means of functions of the system SolidWorks the areas of normal sections are determined, which made $145.02 \mathrm{~mm}^{2}, 144.06 \mathrm{~mm}^{2}, 142.85 \mathrm{~mm}^{2}, 143.15 \mathrm{~mm}^{2}$, $141.89 \mathrm{~mm}^{2}, 138.28 \mathrm{~mm}^{2}, 129.83 \mathrm{~mm}^{2}, 123.28 \mathrm{~mm}^{2}$ and $116.11 \mathrm{~mm}^{2}$. The values of areas of three normal sections $\left(143.15 \mathrm{~mm}^{2}, 141.89 \mathrm{~mm}^{2}, 138.28 \mathrm{~mm}^{2}\right)$ determine the presence of two turning points on the initial diagram of change of normal sections areas of the channel (Fig. 10).

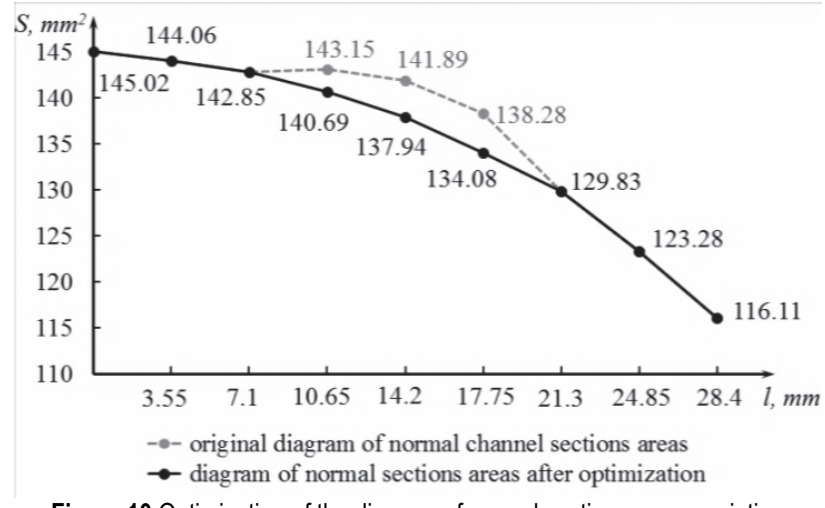

Figure 10 Optimization of the diagram of normal sections areas variation

To provide smooth change of areas the shape of these sections was adjusted. Adjustment is made due to the change of shape of generating line of the impeller's nave. The working surface of the impeller blade was not adjusted. The areas of the changed sections made up $140.69 \mathrm{~mm}^{2}, 137.94 \mathrm{~mm}^{2}$ and $134.08 \mathrm{~mm}^{2}$. The received diagram of change of normal sections areas of the channel is near to parabola.

Optimization of the nave's surface is executed due to the change of the generating line's shape off the surface which is specified in the window of «Property Manager». The changed generating line is formed on the basis of DPC, along which the values of curvature change monotonously. DPC is formed on the basis of nine initial points in which generating line of the initial surface of nave intersects the planes of normal sections of the channel's surface.

Numeration of initial points of DPC corresponds to numeration of normal sections, starting with inlet. Positions of six points $1-3$ and $7-9$ are determined on the border of corresponding normal section of the channel. These borders belong to the surface of the impeller nave.

Positions of points $4-6$ are determined based on the value of area change of corresponding section as a result of optimization.

Position-finding of such point will be considered on the example of point 4, which is assigned on the border of normal section, the area of which makes $137,94 \mathrm{~mm}^{2}$ after optimization.

The method of determination of new border of the optimized normal section is shown in Fig. 10. Adjustment of section's area is realized due to the change of section's border, which belongs to the line on the nave cover. In Fig. 11 it is piece $1-2$. The new section's border is formed $1^{\prime}$ 2 ' equidistance to the initial border $1-2$. The area which line $1^{\prime}-2^{\prime}$ chops off from the initial section (a curvilinear quadrangle $1-2-2^{\prime}-1^{\prime}$ ), equals the difference between initial and optimized sections $\Delta S=141.89-137.94=3.95$ $\mathrm{mm}^{2}$.

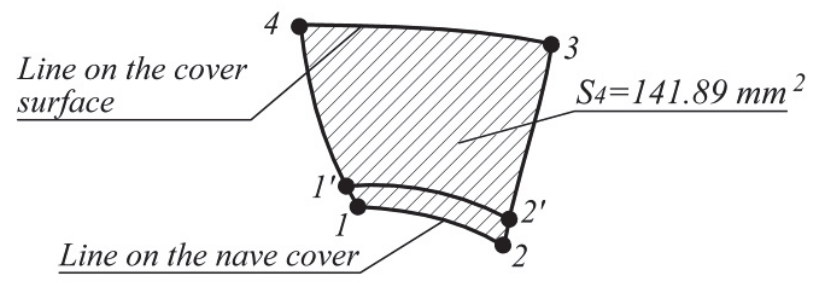

Figure 11 Optimization of the graph of normal channel sections areas variation

Position of the initial point of fourth DPC is determined as a result of crossing of border $1^{\prime}-2^{\prime}$ and the plane which passes through the axis of the nave on the identical distance from points $1^{\prime}$ and 2 '.

On the basis of the set of points, which consists of 9 points, DPC consisting of 56 points is formed. The received points are interpolated by B-spline.

The optimized surface of the impeller nave provides smooth change of normal sections' areas along the channel. Thus the values of areas in nine control sections correspond to the diagram of the areas' change, presented in Fig. 10.

An optimized model of the impeller was designed on the basis of the derived surfaces.

Reanalysis of the flow in the blade channel revealed monotonic pressure growth of the flow lengthwise the channel (Fig. 12).

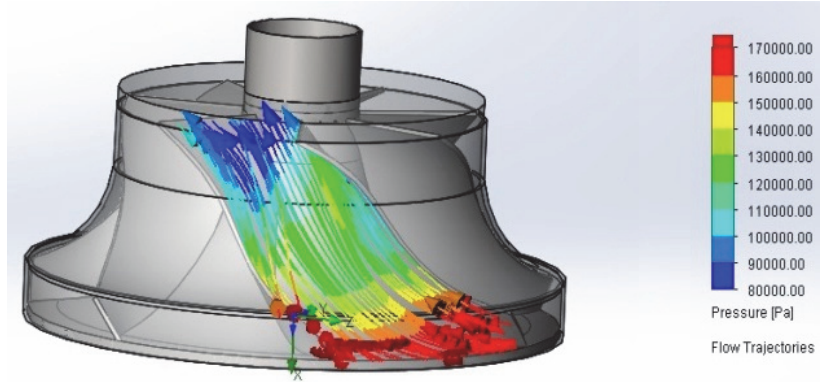

Figure 12 Distribution of pressure inside the flow in the optimized blade channel

Distribution diagram of pressure inside the optimized channel indicated decrease of the flow turbulence.

CAD model of the impeller of the turbine compressor has been designed on the basis of the obtained frame. This model is used as initial data for creation of the program for CNC machine in CAM system of PowerMill. The parts that are manufactured with the $\mathrm{CNC}$ machine passed the tests of the closed loop system.

The tests identified increase of the turbine compressor capacity by $2.5 \%$.

\subsection{Discussion of Results}

The developed method of designing the frame of the surface specified by the array of points includes the following stages:

1. Extraction of the points sets from the array of points. These sets represent plane or space curves (DPC)-initial data for generating elements of the surface frame. Derived 
DPCs are divided into pieces. On their basis it is possible to generate monotonic curves.

2. The points' sets are generated on the basis of initial DPCs via software developed in the system of computer algebra Maple. These sets of points consist of an arbitrary large number of nodes that can be interpolated by curves with specified properties. The ranges of possible values of curvature and torsion are determined in the initial points, at which the forming of the monotonous DPCs is possible. The monotonous pieces of DPC are formed separately.

3. On the basis of initial points and geometrical characteristics assigned in them, the area of possible location of the monotonous curves is determined; with them it is possible to interpolate a set of points.

4. By means of software, worked out in the system of computer algebra of Maple, condensing of the set of points is realized. On the basis of initial set of points a new set of points is generated which consists of arbitrary large number of nodes, which it is possible to interpolate with the curves of set properties. An area is determined of possible location of the monotonous curve which interpolates the received set of points.

5. The contours have been designed in a threedimensional parametric modelling system of SolidWorks on the basis of obtained DPCs. The contours consist of continuous curves' areas, for example, of straight lines' segments, arcs of circles, B-spline. The contour is formed based on the set accuracy. The accuracy of forming the contour is determined by distance from its pieces to the borders of possible location area of DPC.

6. Contours are used as elements of the surface frame. The computer model of the product has been designed on the basis of the frame via standard functions of SolidWorks. The set geometrical characteristics of curves, which with the set accuracy present the linear elements of the frame, provide the necessary functionality of the surface.

Software has been worked out in CAD system of SolidWorks for line generating automatization. The software is developed in the environment of Delphi 10.2., using the tool of API (Application Program Interface). Initial data for the program operation are text files with the positions of points, which are calculated in Maple. The contour which interpolates an initial set of points is created with the use of software in the automated mode. The use of software enables to create the contour in CAD system of SolidWorks, consisting of arbitrary large number of sections.

The peculiarity of our worked out method is generating DPC with successively repeating condensations of a set of points. Thus the area of position of the curve which interpolates the set of points is being inspected step-bystep. The obligatory stage of forming DPC is an analysis that results in determining geometrical characteristics and area of possible location of the monotonous curve, which is possible to use for interpolating the set of points. It provides necessary accuracy with which the contour represents the curve with set geometrical characteristics.

The method allows increasing the number of conditions which are put on the DPC being generated. It can be prevention of oscillation, monotonous change of curvature and torsion along the curve, fixed values of geometrical characteristics in the initial points. Each of additional conditions results in localization of area of possible location of DPC and diminishes an error with which the contour presents it.

Common methods of forming contour (interpolation by second-order curves, by the curves of Bézier, B-spline) [16-19] do not allow to control accuracy of interpolation. An error with which the contour presents initial geometric image can be calculated only after modelling the contour and providing that the initial geometric image is known. Other methods that allow controlling the dynamics of curvature change and torsion along the contours are unknown to us.

Our developed method allows estimating an error, with which the contour is formed by other methods, represents the initial curve with the known geometrical characteristics, with the initial set of points belonging to this curve. The known characteristics of the initial curve will be considered by us as characteristics of the contour being formed, that are possible to be controlled. For example, for flat B-spline it is the control of change of convexity-concavity of the curve, and for space B-spline it is controlling the movement of the curve. Therefore, the error of interpolation by flat B-spline is estimated by the sizes of basal triangle, and interpolation by space B-spline is estimated by the sizes of tetrahedron of location of DPC. Thus, the corresponding tops of triangles or tetrahedrons must coincide with initial points. The methods known to us do not allow decreasing exactness of discrete interpolation due to providing monotonous change of curvature and torsion's values along the contour.

It is reasonable to apply the worked out method for solving the tasks of reverse engineering. Forming of model of the working surface of the turbine impeller blade based on the initial array of points is the example of such task.

The generated model of the impeller has been analyzed via Solid Works Flow Simulation. The gas-dynamic analysis revealed alignment of pressure change of the flow lengthwise the channel and within each of normal sections. We estimated distribution of pressure flow lengthwise the separate normal section with values of minimum, maximal and average pressures. The values of pressures were estimated in regard of the color of the diagram, got in SOLIDWORKS Flow Simulation. The values of the indicated characteristics for nine normal sections of the channel are presented in Tab. 3 .

Table 3 The distribution of flow pressure lengthwise the channel

\begin{tabular}{|c|c|c|c|c|c|c|}
\hline \multirow{2}{*}{$\begin{array}{c}\text { Number of } \\
\text { section }\end{array}$} & \multicolumn{3}{|c|}{ Before optimization } & \multicolumn{3}{|c|}{ After optimization } \\
\hline & $\begin{array}{l}P_{\mathrm{av}} / \\
\mathrm{kPa}\end{array}$ & $\begin{array}{c}P_{\min } / \\
\mathrm{kPa}\end{array}$ & $\begin{array}{c}P_{\max } / \\
\mathrm{kPa}\end{array}$ & $\begin{array}{l}P_{\mathrm{av}} / \\
\mathrm{kPa}\end{array}$ & $\begin{array}{c}P_{\min } / \\
\mathrm{kPa}\end{array}$ & $\begin{array}{c}P_{\max } / \\
\mathrm{kPa}\end{array}$ \\
\hline 1 & 95 & 80 & 122 & 95 & 80 & 122 \\
\hline 2 & 111 & 86 & 127 & 111 & 86 & 127 \\
\hline 3 & 117 & 94 & 131 & 117 & 94 & 131 \\
\hline 4 & 120 & 91 & 136 & 129 & 108 & 137 \\
\hline 5 & 114 & 86 & 140 & 138 & 110 & 144 \\
\hline 6 & 130 & 92 & 154 & 153 & 121 & 161 \\
\hline 7 & 159 & 108 & 168 & 164 & 136 & 172 \\
\hline 8 & 171 & 120 & 178 & 175 & 143 & 181 \\
\hline 9 & 181 & 146 & 195 & 184 & 152 & 203 \\
\hline
\end{tabular}

Fig. 13 represents changing of flow pressures in the $5^{\text {th }}$ normal section of the channel after optimization. 


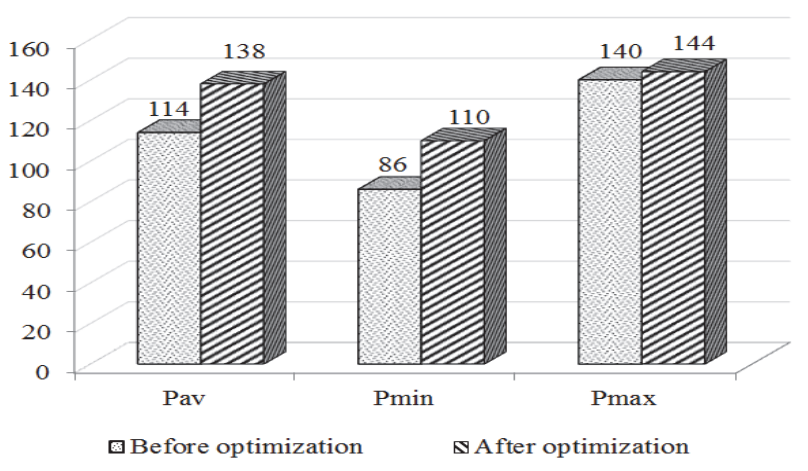

Figure 13 Changing of flow pressures within the normal section of the channel

The improvement of characteristics of the flow inside the blade channel testifies that the dynamic conditions of surfaces, made by the application of the method we suggested, excel the dynamic characteristics of the initial surfaces. Making the impeller by the application of the method that was worked out by us, did not result in the decline of productivity of the turbine compressor.

A basic area of application of the worked out method is a design of dynamic surfaces, including the use of technology of reverse engineering.

The prospect of further development of the method is expansion of its possible application area. Adaptation of the method for solving different tasks can be provided due to the increase in number of conditions which can be imposed on DPC. Such conditions can be increase of number of geometrical characteristics, the values of which are assigned in the initial points, forming of DPC of set length, ensuring touch of DPC with lines or surfaces and others.

The best result can be achieved in solving the tasks, requiring achievement of compromise between the functional characteristics of the surface and additional requirements of arrangement, comfort, and aesthetics.

Development of methods of the contour's forming with the segments of the analytically set curves which are positioned within arbitrary small area of location of DPC, will allow increasing exactness of interpolation.

\section{CONCLUSION}

The method of designing elements of the surfaces' frames on the basis of array of points was suggested in the work. The function of those surfaces is to interact with the environment.

The results of the study are given below.

1. The method of analysis of initial set of point has been worked out. It allows determining areas, which can be interpolated with the monotonous curve. The analysis allows generating the curve on monotonous areas within the area of their possible location. The sizes of area allow estimating an error, with which the formed curve presents a curve belonging to the surface.

2. The algorithm has been developed how to design plane contours that represent the curves with specified geometrical properties with prescribed accuracy: a regular variation values of curvature at minimal number of specific points: points of inflection, points of direction change of increasing of curvature values lengthwise the curve. Flat contours are used in CAD system of SolidWorks as an element of «Profile» at forming the model of surface by means of function of «Lofted Surface».

3. The algorithm has been developed how to design spatial one-dimensional contours that represent the curves with specified geometrical properties with prescribed accuracy: a regular change of curvature and torsion's values at minimal number of specific points: points of trend change, points of direction change of values' increasing of curvature and torsion lengthwise the curve. Space contours are used in CAD system of SolidWorks as an element of «Guide Curves» at forming the model of surface by means of function of «Lofted Surface».

The developed method has been proven while modelling functional surfaces that bound impeller blade channel of a turbine compressor. Quality of forming the model of the impeller is estimated via:

- the gas-dynamic analysis of the flow in the blade channel, which is limited to the surfaces of the impeller and cover of the turbine compressor;

- the tests of the finished product, equipped with the impeller that was made applying our method, on the stand of "closed loop" type.

Tests on the stand and gas-dynamic analysis showed that making of surfaces of the impeller based on the worked out method provided improvement of their dynamic characteristics, compared with those of the initial item.

\section{REFERENCES}

[1] Light, R. \& Gossard, D. (1982). Modification of GeometricModels Through Variational Geometry. Computer-Aided Design, 14(4), 209-214.

https://doi.org/10.1016/0010-4485(82)90292-5

[2] Łukaszewicz, A., Skorulski, G., \& Szczebiot, R. (2018). The main aspects of training in the field of computer-aided techniques (CAx) in mechanical engineering. Proc. of 17 th Int. Scientific Conf. on Engineering for Rural Development, 865-870. https://doi.org/10.22616/ERDev2018.17.N493

[3] Grodzki, W. \& Łukaszewicz, A. (2015). Design and manufacture of unmanned aerial vehicles (UAV) wing structure using composite materials. Materials Science \& Engineering Technology, 46(3), 269-278. https://doi.org/10.1002/mawe.201500351

[4] Mircheski, I., Łukaszewicz, A., Trochimczuk, R., \& Szczebiot, R. (2019). Application of CAx system for design and analysis of plastic parts manufactured by injection moulding. Proc. 18th Int. Scientific Conf. onEngineering for Rural Development. 1755-1760. https://doi.org/10.22616/ERDev2019.18.N463

[5] Sarfraz, M. (2006). Computer-aided reverse engineering using simulated evolution on NURBS. Virtual and Physical Prototyping, 1(4), 243-257. https://doi.org/10.1080/17452750601130492

[6] Salman, M. \& Mansor, A. (2006). Free-form surface models generation using reverse engineering techniques-an investigation. Proceedings of Malaysian Research Group International Conference, 379-385.

[7] Kruth, J. P. \& Kerstens, A. (1998). Reverse engineering modelling of free-form surfaces from point clouds subject to boundary conditions. Journal of Materials Processing Technology, 76(1), 120-127. https://doi.org/10.1016/S0924-0136(97)00341-5

[8] Czerech, L., Kaczyski, R., \& Werner, A. (2012). Machining error compensation for objects bounded by curvilinear surfaces. ActaMechanicaetAutomatica, 6(1), 26-30.

[9] Chovnyuk, Y. V. \& Shutovskiy, O. M. (2016). Analysis of the dynamic behavior of the elastic elements of machines and 
designs: effects of wave generation caused by the motion of the source. Buleten of Kherson National Technical University, 3(58), 164-170.

[10] Steenhuizen, D. \& Tooren, M. I. (2012). The implementation of a knowledge-based framework for the aerodynamic optimization of a morphing wing device. Advanced Engineering Informatics, 26(2), 207-218, https://doi.org/10.1016/j.aei.2012.02.004

[11] Rogerts, D. F. \& Fog, N. G. (1989). Constrained B-spline curve and surface fitting. Computer-Aided Design, 21(10), 641-648. https://doi.org/10.1016/0010-4485(89)90162-0

[12] Carfagni, M., Furferi, R., Governi, L., \& Volpe, Y. (2017). 3D geometry reconstruction from orthographic views: An improved method exploiting shading information. Computers in Industry, 92-93, 137-151. https://doi.org/10.1016/j.compind.2017.07.001

[13] Ivan, M. (2015). A note on the Hermite interpolation. Numerical Algorithms, 69(3), 517-522. https://doi.org/10.1007/s11075-014-9909-x

[14] Argyros, I. K. \& George, S. (2017). On the convergence of Newton-like methods restricted domains. Numerical Algorithms, 75(3), 553-567. https://doi.org/10.1007/s11075-016-0211-y

[15] Liu, S., Chen, Z., \& Zhu,Y. (2015). C1 Rational Quadratic Trigonometric Interpolation Spline for Data Visualization. Mathematical Problems in Engineering, 2015, 983120. https://doi.org/10.1155/2015/983120

[16] Chekalin, A. A., Reshetnikov, M. K., Shpilev, V. V., \& Borodulina, S. V. (2007). Design of Engineering Surfaces Using Quartic Parabolas. IOP Conference Series-Materials Science and Engineering, 221, 012015. https://doi.org/10.1088/1757-899X/221/1/012015

[17] Shen, W., Wang, G., \& Huang, F. (2016). Direction monotonicity of a rational Bézier curve. Applied Mathematics-A Journal of Chinese Universitie, 31(1), 1-20. https://doi.org/10.1007/s11766-016-3399-7

[18] Okaniwa, S., Nasri, A., Lin, H., Abbas, A., Kineri, Y., \& Maekawa, T. (2016). Uniform B-Spline Curve Interpolation with Prescribed Tangent and Curvature Vectors. IEEE Transactions on Visualization and Computer Graphics, 18(9), 1474-1487. https://doi.org/10.1109/TVCG.2011.262

[19] Volkov, Y. S. (2012). Obtaining a banded system of equations in complete spline interpolation problem via Bspline basis. Central European Journal of Mathematics, 10(1), 352-356. https://doi.org/10.2478/s11533-011-0104-1

[20] Barazzetti, L. (2016). Parametric as-built model generation of complex shapes from point clouds. Advanced Engineering Informatics, 30(3), 298-311. https://doi.org/10.1016/j.aei.2016.03.005

[21] Naydush, V. M., Vereschaga, V. M., Naydush, A. V., \& Malkina, V. M. (2007). Fundamentals of Applied Discrete Geometry. Melitopol, M: Lyuks.

[22] Naydush, A. V., Balyuba, I. G., Vereschaga, V. M., \& Spirintsev, D. V. (2018). Variative discrete geometrical modeling. Modern modelling problems, 11, 108-114.

[23] Gavrilenko, E. A. \& Kholodnyak, Y. V. (2014). Discretely geometrical modeling of one-dimensional contours with a regular change of differential-geometric characteristics. Dynamics of Systems, Mechanisms and Machines(Dynamics). 14849712 https://doi.org/10.1109/Dynamics.2014.7005654

[24] Havrylenko, Y., Kholodniak, Y., Vershkov, O., \& Naidysh, A. (2018). Development of the method for the formation of one-dimensional contours by the assigned interpolation accuracy. Eastern-European Journal of Enterprise Technologies, 1(4), 76-82. https://doi.org/10.15587/1729-4061.2018.123921

[25] Hoschek, J. \& Müller, R. (2000). Turbine blade design by lofted B-spline surfaces. Journal of Computational and
Applied Mathematics, $119 \quad(1-2), \quad 235-248$ https://doi.org/10.1016/S0377-0427(00)00381-2

[26] Fooladi, M. \& Foroud, A. A. (2016). Recognition and assessment of different factors which affect flicker in wind turbine. IET Renewable Power Generation, 10(2), 250-259. https://doi.org/10.1049/iet-rpg.2014.0419

[27] Havrylenko, Y. \& Kholodniak, Y. (2014). Formation of geometric model of the impeller of the turbocharger. Proceedings of the Tavria State Agrotechnological University, 14, 48-53.

[28] Kappis, W. (2013). Modern Gas Turbine Systems: High Efficiency, Low Emission, Fuel Flexible Power Generation. Woodhead Publishing Series in Energy, 89-150. https://doi.org/10.1533/9780857096067.2.89

\section{Contact information:}

Yevgen HAVRYLENKO, PhD, associate professor

Department of Information Technologies of Design named after V. M. Naidysh, Tavria State Agrotechnological University,

18 B. Khmelnitsky Ave., 72310, Melitopol, Ukraine

E-mail: yevhen.havrylenko@tsatu.edu.ua

Jose Italo CORTEZ, PhD

Research Laboratory in Digital Systems and Renewable Energy,

Benemerita Universidad Autonoma de Puebla, Mexico,

E-mail: jose.italo@correo.buap.mx

Yuliia KHOLODNIAK, PhD, Senior Lecturer

V. M. Naidysh, Department of Information Technologies of Design,

Tavria State Agrotechnological University,

18 B. Khmelnitsky Ave., 72310, Melitopol, Ukraine

E-mail: yuliya.kholodnyak@tsatu.edu.ua

Hanna ALIEKSIEIEVA, PhD, associate professor

(Corresponding author)

Department of Informatics and Computer Technologies in Management and Learning, Berdiansk State Pedagogical University,

4 Schmidt Str. 71100 Berdiansk, Ukraine

E-mail: alekseeva@ukr.net

Gregorio T. GARCIA, PhD

Research Laboratory in Digital Systems and Renewable Energy,

Benemerita Universidad Autonoma de Puebla, Mexico

E-mail: gtrinidadg4321@gmail.com 